\title{
What we need for COVID-19 post-acute care
}

\author{
Liang-Kung Chen ${ }^{1,2,3}$ (D) Jean Woo ${ }^{4} \cdot$ Hidenori Arai $^{5}$
}

Published online: 2 December 2021

(c) The Author(s), under exclusive licence to European Geriatric Medicine Society 2021

The coronavirus disease 2019 (COVID-19) pandemic has strongly impacted the world for the last 2 years and its related effects will continue despite the development of its vaccines and pharmaceuticals [1-5]. As a novel disease, the pathophysiology leading to respiratory failure and other complications, disease course, and treatment responses of COVID-19 remain unclear, not to mention the long-term effects of severe acute respiratory syndrome coronavirus 2 (SARS-CoV-2) infections and subsequent outcomes of COVID-19 survivors. Growing evidence indicated that COVID-19 involved multiple organ systems in addition to the respiratory system. Carfi et al. reported that $87.4 \%$ of post-acute COVID-19 patients presented with certain symptoms of which fatigue, dyspnea, and joint pain were major leading ones [6]. Another study in China included 1733 patients (median age of 57 years) who survived COVID19 , and found that weakness, sleep difficulties and anxiety or depression remained to be challenges in daily living 6 months after COVID-19 hospitalizations [7]. The "PostCOVID Syndrome" has been proposed to specify persistent symptoms related to residual inflammation, organ damages, and non-specific effects from the hospitalizations or prolonged ventilation, social isolation, or impacts on pre-existing health conditions [8]. Under such circumstances, older adults with multimorbidity, frailty or cognitive impairment

Liang-Kung Chen

lkchen2@vghtpe.gov.tw

1 Aging and Health Research Center, National Yang Ming Chiao Tung University, Taipei, Taiwan

2 Center for Geriatrics and Gerontology, Taipei Veterans General Hospital, No. 201, Sec 2, Shih-ai Road, Taipei, Taiwan

3 Taipei Municipal Gan-Dau Hospital (Managed By Taipei Veterans General Hospital), Taipei, Taiwan

4 Jockey Club Institute of Aging and Department of Medicine and Therapeutics, The Chinese University of Hong Kong, Hong Kong, China

5 National Center for Geriatrics and Gerontology, Obu, Aichi, Japan are especially vulnerable to post-COVID syndrome. A systematic review showed that COVID-19 survivors were highly likely to have impaired physical function, which may be improved by a combined aerobic and resistance training intervention in the post-infectious period [9]. Moreover, neuropsychological symptoms of apathy, executive deficits, impaired cognitive control, and reduction in global cognition have been reported in post-COVID patients, which suggested potential neurological damages by SARS-CoV-2 infections [10]. Based on the above-mentioned conditions, developing post-acute care (PAC) services for post-COVID patients is important, but the nature may differ from current PAC services due to the special pathological characteristics of COVID-19.

In the current issue of European Geriatric Medicine, van Haastregt et al., introduced the EuGMS guidance for the management of post-acute COVID-19 patients [11]. The guidance was developed based on the Dutch guidelines with input from other European countries. Overall, the guidance comprehensively addressed the scenarios of COVID-19 PAC services and recommendations. However, COVID-19 PAC may need to be specifically tailored to target functional and cognitive impairments in post-acute COVID-19 patients. There is still a lack of evidence regarding the syndrome of long COVID in older adults that is crucial for formulating guidelines. Hence, continuous efforts are needed to update the guidance when more clinical data are available. The real challenge to the guidance is whether these services can be effectively provided during the pandemic because of the potential lack of care facilities and resources, as well as the reluctance of older patients and their caregivers to attend hospitals or facilities. More public health policy development and financing designs are needed to tackle the needs for prolonged health challenges specifically related to SARS$\mathrm{CoV}-2$ infections and other common PAC needs subsequent to hospitalizations. The implementation of COVID-19 PAC services also requires successful development of geriatric medicine, although some less well-developed countries may need alternative approaches to cope with the challenges. The United Kingdom Defense Medical Rehabilitation Centre 
published the Stanford Hall Consensus statement for postCOVID-19 rehabilitation that recommended rehabilitation for potential symptoms by organ systems [12]. However, as indicated in the consensus statement, long-term effects of COVID-19 and PAC effects remained uncertain. In contrast to PAC of other conditions, specific infection controls may be needed in COVID-19 PAC services, because 7-23\% of patients who recovered from SARS-CoV-2 infections may show recurrent RNA positivity [13], although it does not always indicate the infectivity of the patient. Therefore, COVID-19 PAC services need to be equipped with standardized infection control measures to avoid potential disease transmission. Moreover, vaccination is another challenge in COVID-19 PAC services, because the timing vaccination of these patients are not well standardized and they are also the vulnerable groups for vaccine-related adverse events [14]. Therefore, the recommendations of the guidance need to be updated to keep pace with the rapidly developing body of knowledge regarding the post-acute phase of the disease.

\section{Declarations}

Conflict of interest All authors declare no conflict of interest.

Ethical approval Tha article does not contain any studies with human participants or animals performed by any of the authors.

Informed consent For this type of study, formal consent is not required.

\section{References}

1. D'Adamo H, Yoshikawa T, Ouslander JG (2020) Coronavirus disease 2019 in geriatrics and long-term care: the ABCDs of COVID-19. J Am Geriatr Soc 68(5):912-917

2. Islam MS, Sarkar T, Khan SH et al (2020) COVID-19-related infodemic and its impact on public health: a global social media analysis. Am J Trop Med Hyg 103(4):1621-1629
3. Noguchi T, Hayashi T, Kubo Y, Tomiyama N, Ochi A, Hayashi $\mathrm{H}$ (2021) Association between family caregivers and depressive symptoms among community-dwelling older adults in Japan: a cross-sectional study during the COVID-19 pandemic. Arch Gerontol Geriatr 96:104468

4. Pranata R, Huang I, Lim MA, Yonas E, Vania R, Kuswardhani RAT (2021) Delirium and mortality in coronavirus disease 2019 (COVID-19) — a systematic review and meta-analysis. Arch Gerontol Geriatr 95:104388

5. Hariyanto TI, Putri C, Arisa J, Situmeang RFV, Kurniawan A (2021) Dementia and outcomes from coronavirus disease 2019 (COVID-19) pneumonia: a systematic review and meta-analysis. Arch Gerontol Geriatr 93:104299

6. Carfî A, Bernabei R, Landi F, Gemelli Against COVID-19 PostAcute Care Study Group (2020) Persistent symptoms in patients after acute COVID-19. JAMA 324(6):603-605

7. Huang C, Huang L, Wang Y et al (2021) 6-month consequences of COVID-19 in patients discharged from hospital: a cohort study. Lancet 397(10270):220-232

8. Garg P, Arora U, Kumar A, Wig N (2021) The, "post-COVID" syndrome: how deep is the damage? J Med Virol 93(2):673-674

9. Rooney S, Webster A, Paul L (2020) Systematic review of changes and recovery in physical function and fitness after severe acute respiratory syndrome-related coronavirus infection: implications for COVID-19 rehabilitation. Phys Ther 100(10):1717-1729

10. Ortelli P, Ferrazzoli D, Sebastianelli L et al (2021) Neuropsychological and neurophysiological correlates of fatigue in post-acute patients with neurological manifestations of COVID-19: insights into a challenging symptom. J Neurol Sci 420:117271

11. van Haastregt JCM, Everink IHJ, Schols JMGA et al (2021) Management of post-acute COVID-19 patients in geriatric rehabilitation: EuGMS guidance. Eur Geriatr Med. https://doi.org/10.1007/ s41999-021-00575-4

12. Barker-Davies RM, O'Sullivan O, Senaratne KPP et al (2020) The stanford hall consensus statement for post-COVID-19 rehabilitation. Br J Sports Med 54(16):949-959

13. Mattiuzzi C, Henry BM, Sanchis-Gomar F, Lippi G (2020) SARS$\mathrm{CoV}-2$ recurrent RNA positivity after recovering from coronavirus disease 2019 (COVID-19): a meta-analysis. Acta Biomed 91(3):e2020014

14. Chen LK (2021) COVID-19 vaccination and frailty in older adults. Arch Gerontol Geriatr 96:104487

Publisher's Note Springer Nature remains neutral with regard to jurisdictional claims in published maps and institutional affiliations. 